\title{
Unexpected Intimacies
}

\section{An Exploration of the Physician-Patient Relationship during the COVID-19 Pandemic}

\section{Kelly Colas}

\begin{abstract}
Aвstract: Anthropologists examining the relationship between physician and patient in Western biomedicine have observed an inherent power discrepancy between the physician, assumed to hold scientific knowledge, and the patient, the recipient of this knowledge. COVID-19 presents a unique challenge to that dynamic, as physicians, scientists and medical experts possess limited understanding of the pathophysiology, interventions and treatment of the disease. Drawing on my experience as a resident physician on the frontlines of the COVID-19 pandemic, I contend that the absence of knowledge surrounding COVID-19 fosters a new form of intimacy between physician and patient through greater emphasis on subjective patient experience, increased transparency between physician and patient, and an expanding physician role beyond management of the physical disease state.
\end{abstract}

KeYwords: biomedicine, COVID-19, intimacy, medical training, moral economy, physicianpatient relationship

Intimacy as a subject of scholarly enquiry provides a useful conceptual tool through which physical, social and temporal relationships can be analysed to glean critical insight to larger cultural and political processes. Yet intimacy is a difficult concept to define, as Ara Wilson succinctly notes: 'Its lack of fixity is part of its appeal' (2012: 32). Rather, intimacy as an analytic frame can be employed to more deeply grasp 'any form and instance of relatedness' and its subsequent impact on relationships in a multitude of sociocultural, political and legal spheres and their intersections with one another (Sehlikoglu and Zengin 2015: 22).

Intimacy is strikingly apparent in the practice of medicine, particularly in the relationship between physician and patient. Often involving the exchange of information considered highly personal, physical touch during exams, and invasive tests and procedures, many of the most vulnerable moments of an individual's life are shared in this encounter.
Yet, social scientists have long observed the nature of this relationship to be asymmetric in its power. Within the dominant system of Western medicine, referred to as biomedicine, the physician is expected to apply scientific data to diagnose and treat a patient, who is viewed as the recipient of this knowledge (Baer et al. 2003; Gaines and Davis-Floyd 2004; Lock and Nguyen 2010). Through the lens of biomedicine, this knowledge is viewed as both objective and certain, and is confirmed through technology such as laboratories and imaging (Clarke and Shim 2003; Delvecchio-Good 2007).

At the end of 2019, a cluster of mysterious respiratory illnesses reported in Wuhan, China, puzzled the scientific community. These cases were later identified as a new viral strain within the coronavirus family called severe acute respiratory syndrome coronavirus 2 (SARS-CoV-2 or COVID-19) (WHO 2020). As COVID-19 cases have surged worldwide, the scientific world has scrambled to better understand this

Anthropology in Action, 27, no. 2 (Summer 2020): 20-27 (C) Berghahn Books and the Association for Anthropology in Action ISSN 0967-201X (Print) ISSN 1752-2285 (Online) doi:10.3167/aia.2020.270203 
disease. Hundreds of millions of dollars have been committed towards research aimed at grasping the pathophysiology of this disease, developing effective interventions, and ultimately creating a vaccine and a successful treatment. Despite these efforts, COVID-19 remains poorly understood.

Less attention has been devoted towards analysing the impact of COVID-19 in the clinical setting, particularly towards how it influences the doctorpatient relationship. This article is my account of working as a frontline internal medicine resident physician during the COVID-19 pandemic. I draw on my experiences working in the COVID-19 intensive care unit (ICU), on COVID-19 hospital wards, and as a resident on the admitting service during the peak of the pandemic in Washington, DC. In this article, I examine the evolving nature of the relationship between doctor and patient in the context of the COVID-19 pandemic, arguing that the uncertainty surrounding COVID-19 distinctly reshapes intimacy between physician and patient.

\section{The Production of Biomedical Knowledge and Relationships}

In order to explore the concept of intimacy as it functions within the physician-patient relationship, it is critical to examine the system that this dynamic unfolds within. Based on the scientific method and heavily reliant on technology, biomedicine views disease as an isolated physical entity (Burri and Dumit 2007; Taylor 2003). Social scientists have questioned the supposed neutrality of this knowledge, highlighting numerous sociopolitical, cultural and economic variables that influence how such information is created and applied, and how it shapes subsequent outcomes (Adams 2016; Bates 2000; Jordan 1993; Sargent and Johnson 1996; Singer 1986).

This seemingly neutral knowledge plays a crucial role in the dynamic between physician and patient. Michel Foucault (1963) famously referred to this dynamic as the 'medical gaze', asserting that the separation of the mind from the body enables physicians to hold power over patients through holding unique knowledge of the body and disease. This power dynamic is in part premised on the assumption that the information applied, based on supposedly objective science, is indisputably true.

Anthropologists remain critical of the assumed certainty of this knowledge. In her essay detailing her experience with cancer in the biomedical system, Susan DiGiacomo writes: 'Beneath the public service of the technologic (and therefore efficacious) biomedicine lies a different reality, one fraught with uncertainty, ambiguity, and contradiction' (1987: 243). DiGiacomo underscores the apparent knowing façade of our medical system, noting that beyond rigid protocols and data, tests and imaging, many questions remain.

This paradox between knowing and uncertainty provides an intriguing window through which intimacy can be examined. Exploring dichotomies like the individual and the collective, the public and the private, and the internal and the external, intimacy can both 'create boundaries as well as flows and transitions between bodies, selves, and groups' (Sehlikoglu and Zengin 2015: 20). Through these fluid interactions, divisions and connections, intimacy enables us to better understand the relationships that are created within a particular social, cultural and political dynamic.

COVID-19 highlights a fundamental tension between science and unpredictability. Most aspects of COVID-19 remain unknown within the biomedical community; scientists presently have a limited understanding of the mechanism of the disease, how to predict the course of the disease, the complications that arise; and any effective interventions for it. COVID-19 is a salient example through which we can explore how the tension inherent in the concept of intimacy unfolds and how it impacts the relationship between physician and patient. In this article, I present my experience as a resident physician in internal medicine during the COVID-19 pandemic at an academic hospital in Washington, DC, asserting that in the context of COVID-19, intimacy in the physician-patient relationship is restructured by this uncertainty.

\section{'It's a Little Different': Subjective Patient Experience in the Context of COVID-19}

The supposedly unbiased nature of biomedicine is often not conducive to incorporating subjective, lived experience (DiGiacomo 1987; Lock and Nguyen 2010). Within the biomedical system, anthropologists have observed that not all forms of knowledge are considered equal. Utilising the term 'authoritative knowledge', Brigitte Jordan (1997) emphasises that the accuracy or correctness of this knowledge is not necessarily important, but rather the significance of this knowledge is that its powerful practitioners consider it to 'count', dismissing all other forms of knowing. In studying the 'formative process' through which medical students learn how to see, speak and present, Byron Good (1993) found that these narra- 
tives reinforce the assumption that biology is at the core of medicine.

However, given the nascence of COVID-19, the knowledge typically considered significant, such as pathophysiology, diagnosis and treatment, is unavailable. Despite increasing recognition of COVID-19 as a serious public health threat, given the limited number of COVID-19 tests, the sole screening criteria in the United States in February 2020 included the following: (1) Has the patient recently returned from Hubei, China; and (2) Did the patient have contact with a known case of COVID-19? In addition to answering 'yes' to one of these questions, the patient also needed the presence of fever, a cough, and shortness of breath in order to undergo testing (CDC 2020). As testing capacity increased, screening criteria gradually expanded to include patients without a travel history or confirmed exposure and to include symptoms like shortness of breath, fever and cough without another plausible explanation (CDC 2020).

Yet, throughout the course of the COVID-19 pandemic, new data and trends thought to be significant emerged. The media and scientific community devoted greater recognition to the large number of asymptomatic carriers of COVID-19 and to less conventional symptoms such as loss of smell and taste, gastrointestinal symptoms, and rashes (Bai et al. 2020; Gu et al. 2020; Landa et al. 2020; Vaira et al. 2020). While rapid COVID-19 testing is now more readily available, scientists question the accuracy of the widely used PCR respiratory swab, noting that tests are prone to operator error and the results may vary depending when in the course of illness the sample was collected (West et al. 2020; Winichakoon et al. 2020). Other diagnostic techniques typically relied upon in the biomedical system such as the physical exam, imaging and blood work may point towards COVID-19, but do not provide a definitive diagnosis of COVID-19.

Given the uncertainty of disease presentation and diagnostic modalities, patients' histories and their descriptions of their experiences gained increasing importance. Below, I will describe a case in which the patient's subjective experience became critical to her medical management:

Early in the COVID pandemic, I admitted a 'frequent flier' patient named Alisha. ${ }^{1}$ She had a host of chronic illnesses that have amounted to a virtual stack of notes from her many hospital admissions. 'This one shouldn't take you more than an hour', a senior resident, familiar with this patient's history, commented. Typically, the reason for her admission was bronchitis on top of her chronic lung disease. Alisha explained that her current symptoms felt very similar to her previous episodes of bronchitis, but that this time she sometimes felt 'a little different'. I continued to pepper her with questions about how she felt different, but she responded that she 'couldn't put her finger on it'. Towards the end of our encounter, the patient mentioned that two members of her family were currently in the ICU for COVID-19, although she was never directly in contact with either of them. Interestingly, Alisha's COVID-19 test had been negative twice now. When I presented this case to the attending physician, he paused for a moment, deliberating whether we should admit this patient as a suspected COVID-19 case and continue precautions despite the hospital's dwindling personal protective equipment (PPE). 'We can't risk it', he concluded. She was ultimately admitted to a ward in the hospital that provides a more advanced level of care and was continued on contact/droplet precautions.

Through the biomedical lens, ambiguous descriptions like 'something is a little different' are typically considered irrelevant details not worthy of documentation or mention to the attending physician. Yet in Alisha's case, despite a negative test, her subjective experience that something was 'different', along with questionable contact with a COVID-19 patient, changed her medical management. Given the lack of knowledge about the presentation and management of COVID-19, the patient narrative gained new importance in deepening our understanding of COVID-19. Unconventional symptoms, employment, living situations and subjective experiences became noteworthy details that we needed to gain a deeper understanding of this disease and its effect on patients.

While undoubtedly the intent of some of the expanded patient narratives is to gather diagnostic clues, it also clears new space to listen to patients' lived experiences. The medical system is structured in a way that even the most well-intentioned physicians feel pressured to filter through patient narratives to find the 'important' information. This is most clearly highlighted on the forms we are given to evaluate medical students; one of the criteria for a high-performing medical student is one that possesses 'superior organization' of information and 'always includes important information'.

The experience of admitting COVID-19 patients will likely continue to evolve as new information emerges and improved diagnostic modalities are available. Yet, in the current environment of uncertainty, COVID-19 has restructured the idea of what information is considered noteworthy. Given our 
limited interpretation from the information that can be gleaned from physical exam findings, tests and imaging in regards to COVID-19, closer listening and a greater emphasis on the subjective patient experience becomes critical.

\section{Uncertain Trajectories: The Implications of Increased Transparency on Physician- Patient Intimacy}

The separation of subjective narrative from supposedly objective clinical data illustrates a defining belief of biomedicine; disease is reducible to a physical entity, free from social, cultural and political influence (Baer et al. 2003; Clarke and Shim 2003). This view of disease as an isolated physical phenomenon also serves to create a distinct power dynamic between physicians and patients, as physicians unilaterally maintain access to this information. However, social scientists deconstruct the assumption that biomedical knowledge is definitive and correct, contending that despite the presentation of certainty, many unanswered questions and great unpredictability exist (Adams 2016; Biruk 2012).

Uncertainty is closely entwined with the concept of intimacy. Lauren Berlant writes: "'I didn't think it would turn out this way" is the secret epitaph of intimacy' (1998: 281). She observes that fundamental to intimacy is the tension between the shared assumption of how relationships exist within a particular institution or framework and the reality that unpredicted events can disrupt these expectations at any moment.

The lack of knowledge around COVID-19 provides an interesting backdrop to examine how this tension unfolds and how it impacts the relationship between physician and patient. Below, I will explore two different experiences of uncertainty, arguing that this ambiguity leads to increased transparency between physician and patient, which differently impacts the actors in play:

I met James, an African American male in his sixties, during my time as the intern responsible for admitting patients to the hospital. James was sitting on the edge of his bed in the emergency room (ER) with a nasal cannula in place; a flimsy hospital gown covered over his top half, he was still wearing his slacks from work underneath. James was summoned to DC from his home state for work; he carefully opted for a nine-hour drive over flying to minimize his COVID-19 exposure. Two hours away from arriving in DC, he began to feel short of breath. 'It's noth- ing', he thought to himself, 'just letting all of this COVID talk go to my head'. By the time he reached DC, he rerouted his GPS to the hospital. In the ER, he desaturated to 80 per cent on room air while walking, requiring oxygen through nasal cannula and a hospital admission.

I spoke to James in a soft shout over the billowing sounds of his negative pressure room and through my N95 mask and face shield. He spoke slowly, taking deep breaths between sentences. After a few minutes, he looked at me directly in the eyes, asking: 'Doc, be straight with me. How is this going to go?' I paused for a moment, searching for the words to answer his question.

'James, I want to be honest with you', I said, locking eyes with him through my face shield. 'I really don't know. Some people just need a little oxygen and rest and they're better within a few days. Others ... have a harder time. Sometimes they need a breathing tube and develop other complications. We don't know which way things will go and why they do'. Silence fell for several seconds. I shifted uncomfortably in my PPE, wondering if I had said the right thing. 'Thank you', James responded, placing his hand over my gloved one, 'I appreciate the honesty'.

By the time I arrived at the hospital the next day, James' oxygen requirement had escalated so quickly overnight that he was now intubated in the ICU. I thought of my experience with James frequently throughout the pandemic. I previously felt uncomfortable with admitting any lack of knowledge; the concept of 'knowing' is so deeply ingrained into medical education and training. Even if we are unsure of the exact diagnosis or treatment, the expectation is to at least have the skills to gather the clinical clues to determine the diagnosis.

This encounter with James was wrought with mutual uncertainty, but our positions differed. One of the fundamental assumptions underlying biomedicine is that the patient does not know or understand their illness, but the physician is able to uniquely know, interpret and treat. Yet, in the context of COVID-19, there was little information or knowledge that the biomedical system could provide. James acknowledged this lack of knowing and the unpredictability that came along with it.

However, the heightened transparency around the uncertainty of COVID-19 was met with resistance. As I will illustrate in the example below, discussing the capricious nature of COVID-19 with family members sometimes resulted in distrust in our relationship with them:

Juan is a previously healthy man in his sixties whose ICU stay was extended by months due to COVID-19. 
Juan was still was unable to breath on his own, requiring the support of the ventilator. Furthermore, Juan's mental status substantially declined during his course of COVID19. He was completely unresponsive; he no longer tracked with his eyes, withdrew from pain, or interacted in any way.

I spoke on the phone with his wife, daughter and son daily. 'How's his mental status?' his son asked eagerly. Each day, I delivered the unfortunate news that there had been no changes. They pressed as to why this was. I told them that his poor mental status was due to acute metabolic encephalopathy, meaning that some sort of infectious, toxic, or metabolic imbalance in the body caused altered mentation. In Juan's case, this was likely caused by lack of oxygen in conjunction with systemic infection. I explained that we are still learning all of the ways that COVID-19 can variably affect different organ systems, and it is possible that Juan's COVID-19 infection was impacting his mentation in a way that we have yet to understand.

The patient's son scoffed at this explanation. 'Nah, there must be something else. This isn't like him', he son decided. 'He's a fighter, he'll turn around'. Juan's wife expressed a similar view, continually pressing about different diagnostic modalities to know the cause of his altered mental status. 'Surely there must be a different scan or blood test', his wife reasoned, 'I think that you're missing something'.

As Juan's course progressed, his son began to suggest experimental treatments and a 'miracle drug' that they had heard about on the news. 'Something will work; you need to do more', he urged.

Juan's family grappled with his unclear diagnosis and prognosis and lack of treatment. Despite our best efforts to transparently relay Juan's clinical condition to his family, they found this change in his health to be inconceivable. Two months ago, he was jogging and making dinner for his family. They struggled to believe the rapid deterioration of his clinical status and to accept the ambiguity underlying his condition. Our admission that we could not fully explain why Juan's condition continued to decline without any clear, efficacious treatments worsened the trust between Juan's family and our team, as they questioned the commitment of the medical team to 'do more'.

Juan's and James' situations diverge in several ways. First, it is critical to note that the encounter with Juan occurred further along in his illness. Uncertainty as to whether he would improve still remained, although at this stage there was overwhelming evidence to suggest that he would not. Conversely, while James was seriously ill, at the point in time that I met him it remained unknown whether he would quickly improve or decline. The time at which these encounters respectively occurred may have shaped the response to the degree of uncertainty surrounding their prognosis.

It is also critical to consider the context in which both of these situations occurred. James was in the ER; he felt his symptoms and he knew something was not right. He saw the hospital staff donning our $\mathrm{PPE}$, continually running in and out of rooms of very ill patients.

Due to the hospital policy barring visitors, Juan's family was limited to video chat for a handful of minutes a day. In discussing Juan's case with the critical care attending physician, she expressed feeling that the situation with Juan's family was a familiar experience to her during COVID-19. 'There's no trust there', she commented. 'Without these families coming in to the hospital, they're not seeing this. They don't experience the ICU. They don't meet our team and see everything that we're doing'. The discrepancy between the expectation and reality of COVID-19 is likely exacerbated by patients' families' inability to witness the situation and see their loved one, especially as the media and American political leaders deliver conflicting messages about potential interventions and treatments.

While transparency regarding the uncertainty of COVID-19 can enhance intimacy between physician and patient in the context of the hospital, the lack of answers can also result in greater distrust. In situations such as James', the recognition of the unpredictability of COVID-19 fostered vulnerability, increasing the relatedness between us. Conversely, the admission of the uncertainty of the manifestations, prognosis and treatment of COVID-19 also generated suspicion, confusion and doubt.

\section{Moral Economy and Medical Needs: Expanding the Role of the Biomedical Physician}

A hallmark of biomedicine is the reduction of the illness experience to the physical disease entity. While medical school curriculums have attempted to incorporate holistic elements of patients' well-being in their care such as 'cultural competency' courses or assessments on bedside manner, the focus on disease pathophysiology, management and treatment composes the bulk of medical training (Kleinman and Benson 2006). Additionally, focussing on needs apart from physical ones becomes increasingly difficult as 
physicians manage an overwhelming patient load, complete extensive documentation, and work within the restrictions of health insurance (Hunt et al. 2017; Oldani 2010).

Yet, COVID-19 provides a challenge to the biomedical belief that physicians' role is primarily disease management, given the lack of interventions and treatments available. The function of biomedicine in COVID-19 is primarily supportive, including aiding patients' breathing with supplemental oxygen, trialling breathing treatments, and managing any other complications that arise. Additionally, hospital policies restricting any visitations from patients' family and friends have left a void for emotional support and advocacy.

Throughout the COVID-19 pandemic, I was continually inspired by the kindness and attentiveness that I witnessed in my colleagues' care for their patients. One of my colleagues brought a personal iPad for 'music rounds', spending time each day playing the favourite music of patients who were sedated and intubated in the ICU. Another colleague described the experience of holding a patient's hand after he was extubated and ultimately passed away while comforting the patient's family on video during this process. Many of my co-residents spent additional hours after already long and gruelling shifts facetiming and updating patients' families.

These acts of focussing on patients' non-medical needs were also apparent outside of the COVID-19 ICU. Several of my co-workers spent substantial portions of their day embarking on tasks like finding battery replacements for hearing aids, taking patients on walks through the hallway, or simply chatting with patients in their rooms.

A number of social scientists have observed that a challenging or uncertain environment provides an intriguing backdrop through which we can examine the renegotiation of beliefs and actions. The concept of 'moral economy' is a useful theoretical tool that can be applied to describe the shared mores and values with which people evaluate their relations with others' (Prince 2012: 536). For example, in Clare Wendland's (2012) examination of Malawian medical students, she found that young physicians navigated the injustice and poverty that they encountered by developing a 'moral map', a perspective of their environment that creatively influenced their management of patients. Wendland found that students developed greater physical exam skills in the absence of technology and largely blamed structural problems for patients' health disparities, rather than the patients themselves. Similarly, Robin Higashi and colleagues
(2013) illustrated that medical trainees reshape their 'moral economies' in working with 'difficult' patient populations, finding meaning and fulfilment in patient encounters assumed to add little value to their time and education.

The concept of 'moral economy' applied to biomedicine during the time of COVID-19 is helpful in illustrating the shifting role of health-care providers. Without clear medical treatments, constantly changing public health recommendations, and the absence of family and patient advocates, physicians' focus is broadened beyond managing physical disease. Increasing attention devoted towards aspects of patients' well-being that are traditionally considered by biomedicine to be 'non-medical' provides a sense of meaning amidst a lack of interventions and treatments.

Additionally, I found that physicians' perspectives and care of patients was influenced by a sentiment that a co-resident expressed in her response to an e-mail from hospital administration that claimed that 'we are all in it together'. Rolling her eyes, she quipped: 'We [health-care workers and patients] are in this together. They [hospital administration] couldn't be less in it'. As a resident on the admitting service when the pandemic first began to escalate in Washington, DC, our frustration began to quickly grow at the hospital's rapidly changing PPE guidelines, the difficulty of obtaining proper PPE, and the administration's unwillingness to be transparent about the hospital's PPE supply. Furthermore, escalating acknowledgement of an abysmal public health response by the United States government and growing recognition of the devastatingly disproportionate impact of COVID-19 on poor, minority groups created a sense of solidarity and commitment to caring for our patients in any way possible.

\section{Conclusion}

Across the anthropological study of intimacy, scholars note that a distinct characteristic of intimacy is its ability to create unexpected relationships, bonds and relatedness. Berlant writes: 'Intimacy builds worlds; it creates spaces and usurps places meant for other kinds of relation' (1998: 282). Following Berlant's assertion that intimacy creates new relations in spaces intended for other purposes, I argue that while the biomedical encounter is historically characterised by a unilateral transfer of knowledge from physician to patient, the ambiguity of COVID-19 reframes this dynamic. The uncertainty surrounding COVID-19 restructures intimacy in the physician-patient rela- 
tionship by placing greater emphasis on subjective experience, creating greater transparency between physician and patient, and redefining physicians' roles beyond strict medical management. COVID-19 underscores the limitations of biomedical knowledge and diminishes the subsequent division between physician and patient.

Intimacy as a theoretical tool enables a more profound understanding of how connections, relatedness and meaning develop within a larger institutional, political and social framework. As the COVID-19 pandemic continues to evolve, it remains critical for social scientists to explore the interplay of social, cultural and political variables and their impact on daily relationships and interactions, and to observe the power structures that are disrupted, shifted or magnified.

\section{Acknowledgements}

I would like to thank my co-residents for continually inspiring me with their compassion and tireless efforts. I am also grateful to the patients and families that I have had the privilege to care for over this year for teaching me deeper empathy, humility and kindness. Finally, I must thank my friends and family; this work would not have been possible without their unwavering support and limitless encouragement.

Kelly Colas received a PhD in Anthropology from Michigan State University in 2017 and graduated from Michigan State University's College of Osteopathic Medicine in 2019. She is currently a secondyear resident physician in internal medicine in Washington, DC. Her research interests include health disparities, access to care, physician-patient relationships, and health care in Latin America.

E-mail: Kelly.colas@medstar.net

\section{Note}

1. All patient names have been changed for privacy reasons.

\section{References}

Adams, V. (2016), Metrics: What Counts in Global Health (Durham, NC: Duke University Press).
Baer, H. A., Merrill Singer and Ida Susser (2003), Medical Anthropology and the World System (Westport, CT: Greenwood Publishing Group).

Bai, Y., L. Yao, T. Wei, F. Tian, D.-Y. Jin, L. Chen and M. Wang (2020), 'Presumed Asymptomatic Carrier Transmission of COVID-19', JAMA 323, no.14: 14061407, doi:10.1001/jama.2020.2565.

Bates, D. G. (2000), 'Why Not Call Modern Medicine "Alternative"?' Perspectives in Biology and Medicine 43, no. 4: 502-518, doi:10.1353/pbm.2000.0032.

Berlant, L. (1998), 'Intimacy: A Special Issue', Critical Inquiry 24, no. 2: 281-288, doi:10.1086/448875.

Biruk, C. (2012), 'Seeing Like a Research Project: Producing "High-Quality Data" in AIDS Research in Malawi', Medical Anthropology 31, no. 4: 347-366, doi :10.1080/01459740.2011.631960.

Burri, R. V., and J. Dumit (2007), Biomedicine as Culture: Instrumental Practices, Technoscientific Knowledge, and New Modes of Life (New York: Routledge).

Centers for Disease Control (CDC) (2020), 'CDC Overview of Testing for SARS-CoV-2', 2 July, https:// www.cdc.gov/coronavirus/2019-ncov/hcp/testingoverview.html.

Clarke, A., and J. K. Shim (2003), 'Medicalization and Biomedicalization Revisited: Technoscience and Transformations of Health, Illness and Biomedicine,' American Sociological Review 68, no. 2: 161-194, doi:10.2307/1519765.

Delvecchio-Good, M. J. (2007), 'The Medical Imaginary and the Biotechnical Embrace: Subjective Experiences of Clinical Scientists and Patients,' in Subjectivity: Ethnographic Investigations, (eds.) J. Biehl, B. Good, and A. Kleinman (Berkeley: University of California Press), 362-380.

DiGiacomo, S. M. (1987), 'Biomedicine as a Cultural System: An Anthropologist in the Kingdom of the Sick: Encounters with Biomedicine', in Case Studies in Medical Anthropology, (ed.) H. A. Baer (New York: Gordon and Breach Science Publishers), 315-346.

Foucault, M. [1963] (2012), The Birth of the Clinic, (trans.) A. M. Sheridan (London: Routledge).

Gaines, A. D., and R. Davis-Floyd (2004) 'On Biomedicine', in Encyclopedia of Medical Anthropology, (eds) C. Ember and M. Ember (New York: Kluwer Academic), 95-109.

Good, B. J. (1993), Medicine, Rationality and Experience: An Anthropological Perspective (Cambridge: Cambridge University Press).

Gu, J., B. Han and J. Wang (2020), 'COVID-19: Gastrointestinal Manifestations and Potential Fecal-Oral Transmission', Gastroenterology 158, no. 6: 1518-1519, doi.org/10.1053/. 
Higashi, R. T., A. Tillack, M. Steinman and B. Johnson (2013), "The "Worthy" Patient: Rethinking the "Hidden Curriculum" in Medical Education', Anthropology E Medicine 20, no. 1: 13-23, doi:10 .1080/13648470.2012.747595.

Hunt, L. M., H. S. Bell, A. M. Baker and H. A. Howard (2017), 'Electronic Health Records and the Disappearing Patient', Medical Anthropology Quarterly 31, no. 3: 403-421, doi:10.1111/maq.12375.

Jordan, B. (1993), Birth in Four Cultures: A Crosscultural Investigation of Childbirth in Yucatan, Holland, Sweden, and the United States (Prospect Heights, IL: Waveland Press).

Jordan, B. (1997), 'Authoritative Knowledge and Its Construction', in Childbirth and Authoritative Knowledge: Cross-Cultural Perspectives, (eds) R. D. Floyd and A. C. Sargent (London: University Of California Press), 55-79.

Kleinman, A., and P. Benson (2006), 'Anthropology in the Clinic: The Problem of Cultural Competency and How to Fix It', PLoS Med 3, no. 10: e294, doi: 10.1371/journal.pmed.0030294.

Landa, N., M. Mendieta-Eckert, P. Fonda-Pascual and T. Aguirre (2020), 'Chilblain-Like Lesions on Feet and Hands during the COVID-19 Pandemic', International Journal of Dermatology 59, no. 6: 739-743, doi:10.1111/ijd.14937.

Lock, M., and V. K. Nguyen (2010), An Anthropology of Biomedicine (Chichester, UK : Wiley-Blackwell).

Oldani, M. J. ( 2010), 'Assessing the "Relative Value" of Diabetic Patients Treated through an Incentivized, Corporate Compliance Model', Anthropology $\mathcal{E}$ Medicine 17, no. 2: 215-228, doi:10.1080/13648470.20 10.493649.

Prince, R. (2012), 'HIV and the Moral Economy of Survival in an East African City', Medical Anthropology Quarterly 26, no. 4: 534-556, doi:10.1111/ maq.12006.
Sargent, C. F., and T. M. Johnson (1996), Medical Anthropology: Contemporary Theory and Method (Westport, CT: Praeger).

Sehlikoglu, S., and A. Zengin (2015), 'Introduction: Why Revisit Intimacy?' Cambridge Journal of Anthropology 33, no. 2: 20-25, doi:10.3167/ca.2015.330203.

Singer, M. (1986), 'Developing a Critical Perspective in Medical Anthropology', Medical Anthropology Quarterly 17, no. 5: 128-129, doi:10.1111/j.1937-6219.1986 .tb01055.x.

Taylor, J. (2003), 'Confronting "Culture" in Medicine's "Culture of No Culture"', Academic Medicine 78, no 6: 555-559, doi:10.1097/00001888-200306000-00003.

Vaira, L. A., G. Salzano, G. Deiana and G. De Riu (2020), 'Anosmia and Ageusia: Common Findings in COVID-19 Patients', The Laryngoscope 130, no. 7: 1787, doi:10.1002/lary.28692.

Wendland, C. (2012), 'Moral Maps and Medical Imaginaries: Clinical Tourism at Malawi's College of Medicine', American Anthropologist 114, no. 1: 108-122, doi:10.1111/j.1548-1433.2011.01400.x.

West, C., V. M. Montori and P. Sampathkumar (2020), 'COVID-19 Testing: The Threat of False-Negative Results', Mayo Clinic Proceedings 95, no. 6: 11271129, doi:10.1016/j.mayocp.2020.04.004.

Wilson, A. (2012) 'Intimacy: A Useful Category of Transnational Analysis', in The Global and the Intimate, (ed.) G. Pratt and V. Rosner (New York: Columbia University Press), 31-56.

Winichakoon, P., R. Chaiwarith, C. Liwsrisakun, P. Salee, A. Goonna, A. Limsukon and Q. Kaewpoowat (2020), 'Negative Nasopharyngeal and Oropharyngeal Swabs Do Not Rule Out COVID-19', Journal of Clinical Microbiology 58, no. 5: e00297-20, doi:10.1128/ JCM.00297-20.

World Health Organization (WHO) (2020), ‘WHO Timeline-COVID-19', https://www.who.int/ news-room/detail/29-06-2020-covidtimeline. 